\title{
Footstep Pressure Signal Analysis for Human Identification
}

\author{
Michael Edwards and Xianghua Xie \\ Department of Computer Science, \\ Swansea University, Swansea, SA2 8PP, United Kingdom \\ http://csvision.swan.ac.uk
}

\begin{abstract}
Pressure derived footstep signals are a growing field in biometrics, offering unobtrusive sample collection in comparison to established biometrics, with strong classification accuracy despite the highly variable nature of input instances. As a weak biometric, footsteps obtain lower predictive accuracy than stable alternatives and real world implementation will require reliable, yet flexible, feature sets that enable accurate class label partitioning. We suggest a method of retaining the spatial dimensions that are commonly lost during production of ground reaction force profiles and demonstrate the use of wavelet analysis on raw pressure signals for feature production. By analysing pressure signals obtained from common piezoelectric sensor arrays, we have trained a Random Forest classifier for individual prediction within a dataset of 10,413 footstep pair instances from 94 participants. Retaining spatial information for wavelet analysis returned error rates as low as $16.3 \%$, showing strong predictive accuracy on a large, natural dataset.
\end{abstract}

\section{INTRODUCTION}

Biometric measures have proven to be a popular method for individual identification, with multiple biometric parameters drawing the focus of extensive research [1]. Suitability of parameters for use as classification markers is based on intraand inter-individual variability of the metric in question; a need to accurately distinguish between two individuals, while flexibly classifying multiple input instances from the same individual. Biometrics are often divided into two groups; physiological biometrics, based on stable physical characteristics, and behaviometrics, dependant on more flexible behaviour. Physiological biometrics such as fingerprint, iris pattern and DNA sequences are well researched and, with suitable feature extraction, show high classification accuracy [2]. Behaviometrics such as gait and speech pattern are more variable in their usage as an identifier, with some parameters showing high accuracy whilst others are still in the early stages of evaluation. Works such as pose extraction [3] and interaction modelling [4] are often necessary for deriving those behaviometrics. Footsteps as a behaviometric is relatively young topic, with literature suggesting their use in multi-modal systems as an auxiliary identifier, due to lower classification accuracy in comparison to their established counterparts [5], [6]. As such, footsteps are often classified as a weak biometric due to high intra-individual variability between multiple footstep instances and potential for external impact on inter-individual behaviour. Despite this concern, pressure based footsteps have shown to provide reasonable classification even with masking of the foot silhouette by footwear or added weight loading
[7], [8], suggesting pressure collection apparatus can benefit over image based biometrics, which suffer during occlusion scenarios, and provide minimal obstruction to the user during sample collection with sensors providing little physical barrier to user movement.

The ability of biometrics to seemingly identify individuals makes them an attractive choice as authentication tools in security systems or personalised system interaction. Biometric classifier systems are often designed for verification, determining if a sample belongs to approved users or should be rejected; this differs from the identification problem, in which the sample is classified to one label out of many. To partition a set of class labels, semi-supervised classifiers utilise a feature set that represents the individual in question, such that new inputs can be compared against enrolled users to determine correct class. Footstep biometric systems often utilise representative profiles that condense either the spatial or temporal domain from raw data, from which features are extracted and used to train the classifier. The most common profile is the Ground Reaction Force (GRF), providing a description of force from the foot imparted on the floor during a step [8]-[12]. Using GRF features detailed by [11] it has been possible to achieve classification accuracy rates of $93 \%$ when classifying users with nearest-neighbour based recognition, [8], and $>95 \%$ when combining multiple classifiers with a rejection class on a dataset consisting of 10 participants, [11]. These high classification rates are often obtained using small datasets, extensive classifier tuning or by sample verification as opposed to classification; issues which may result in diminished accuracy when applied to real world implementation. Therefore we intend to utilise a large and highly variable dataset to train a classifier to predict the identity label of an input sample, providing a more realistic interpretation of implementation.

Extraction of suitable features are key to the production of accurate classifiers and use of continuous wavelet transform (CWT) as a signal-processing tool has allowed identification of signal events in both time and frequency domains. This offers benefit over the Fourier transform when temporal location of events is important, most notably during inconsistent or zero-averaging signals; such as those presented within footstep pressure channels. Current CWT analysis of footstep signals focuses on gait analysis of multiple consecutive footsteps [13] and foot topography estimation [6]. Wavelet transform has 
most commonly been used in the analysis of audio signals obtained from footfall during the step using microphone [14], [15] or the measurement of ground vibrations using a geophone [16], [17]. The discrete form of the wavelet transform is often used to correct signals by smoothing out background measurement noise [16], [18]. Despite the study of wavelet uses in the extraction of features from audio and image signals, little has been done in analysis of GRF profiles formed from simple footstep pressure collection, and even less has been studied on the use of wavelets to analyse the raw pressure signals from a multi-sensor array. In [7], the authors found that the use of wavelet analysis on single step GRF profiles improved prediction accuracy over use of more common heuristic features, which suggests the use of CWT as a signal analysis tool can be effective for event localisation within footstep instances. It has also been shown by [19] that the use of wavelet transforms can be used to classify individuals with knee replacements in a clinical setting

The lack of wavelet analysis on raw footstep pressure signals has led this study to apply CWT to the raw data signals obtained by pressure sensitive mats. We intended to retain spatial information lost during GRF profile production and draw upon the strength of wavelet based signal analysis in order to more accurately identify individual participants. The GRF profile is often used to remove the spatial information from the footstep to reduce it into a simplified pressure/time sequence [8]. The spatial information within a footstep can be a defining parameter; with the morphology and size of the foot being used as a classification feature in image based approaches. Such consideration of the spatial domain in the imaging methods have allowed high accuracy rates to be achieved and this study aims to show that the same consideration can be applied to the pressure signal methodologies. By retaining the spatial domain we hope to show that the spatial nature of the footstep is important to its use in determining classifiable features, and as such the overall spatio-temporal footstep should be considered as a whole.

We also explore the use of a Random Forest classifier in prediction of individual identity using foot-floor impact based pressure data. A mixture of statistical geometric features drawn from raw signals, representative profiles and wavelet analysis of raw data will be used to construct feature sets for classifier training. These features are then used to train the classifier in an attempt to predict individual class labels. Previous study has made use of the global GRF feature, which condenses several channels into one profile, resulting in a loss of spatial domain information; as such we intend to retain spatio-temporal information within raw channels for analysis and feature production, while comparing against the use of geometric profile features and wavelet analysis of the common GRF profile.

\section{Proposed Method}

Below we outline the method taken in collection of pressure based footstep profiles, extraction of geometric and wavelet processing feature sets and construction of a Random Forest
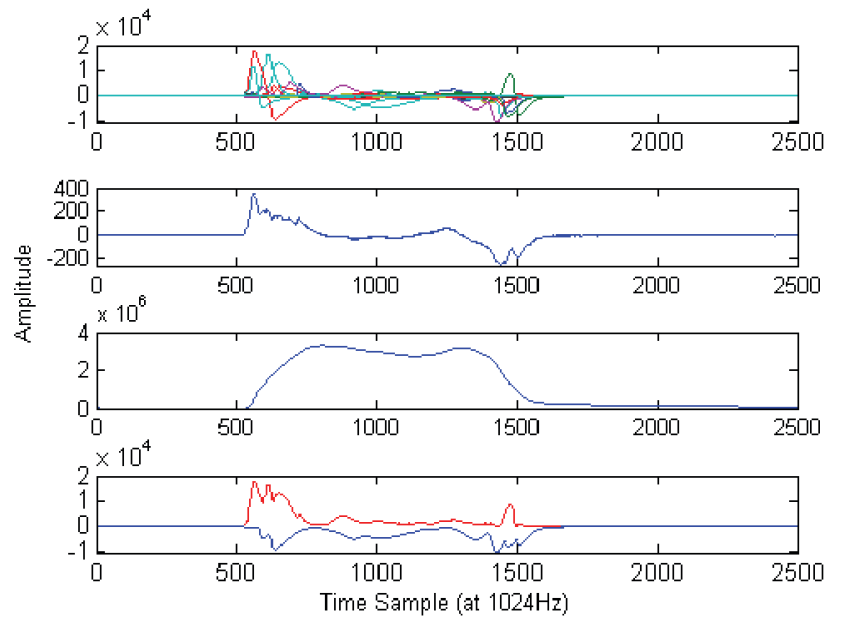

Fig. 1: Footstep profiles, from top: raw pressure, $S_{\text {ave }}$, GRF and contours (red: upper contour, blue: lower contour).

classifier to predict class labels. We discuss the use of basic statistics drawn from footstep raw data and representative profiles, continuous wavelet transform as a processing tool for analysis of GRF footstep profiles and our proposed method of retaining spatial information via wavelet transform of raw signals. For all analysis we consider the footstep to consist of both left and right foot placement instances, using both feet as one sample has been shown to provide higher predictive accuracy, as opposed to using individual feet [8].

\section{A. Data}

The Swansea University Speech and Image Research Group footstep dataset of over 11,000 pressure based footstep samples was used to develop a training/testing set [20]. Each sample was collected, at a sampling rate of $1024 \mathrm{~Hz}$, by the traversal of two $45 \times 35 \mathrm{~cm}$ piezoelectric sensor mats placed within a doorway. This produced a left and right combined footstep profile consisting of 88 channels per foot (top Fig.1). Erroneous samples, such as incomplete mat traversal and directional inconsistency, were removed from the dataset. To conform to the 10-fold cross validation requirements, classes with $<10$ samples were removed. Preening of the collection provided a final dataset of 10,713 samples taken from 94 individual participants, with participants providing a range of samples, from $<20$ to in excess of 1,000 footstep instances. Participant inputs were subject to varying footwear, loads and walking speeds, providing a natural dataset of highly variable samples. Feature sets, discussed below and summarised in TableI, were derived from the dataset to allow classifiers to be constructed.

\section{B. Geometric features}

Using common methodology in footstep analysis, raw amplitude/time signals were transformed into a set of representative profiles, Fig.1, which were used to draw a set of geometric feature vectors. These profiles are found throughout literature 
TABLE I: Feature sets for classifier construction.

\begin{tabular}{ll}
\hline Feature & Description \\
\hline \hline Geometric & $\begin{array}{l}\text { Statistics drawn from raw data, } \mathrm{S}_{\text {ave }}, \mathrm{GRF} \text { and contour } \\
\text { profiles } \\
\text { Multi scale GRF step lengths }\end{array}$ \\
\hline Wavelet & $\begin{array}{l}\text { Multi wavelet analysis of GRF profile } \\
\text { Wavelet analysis of raw pressure signals retaining the } \\
\text { spatial domain }\end{array}$ \\
\hline Proposed & $\begin{array}{l}\text { Aggregation of geometric, GRF wavelet analysis and } \\
\text { spatially-retentive wavelet analysis }\end{array}$ \\
\hline
\end{tabular}

concerning energy distribution within a footstep sample, with the GRF being a successful focus of study [7], [8]. These geometric profiles allow information contained in multiple channels to be considered as a single entity, by reducing sample dimensionality through methods such as spatial averaging, $\mathrm{S}_{\text {ave }}$ (Fig.1 second from top), cumulative effect, GRF (Fig.1 third from top) or raw data contouring, (Fig.1 bottom).

The global GRF, frequently found across literature [7], [10], [11], [13], [21], presents the overall pressure change across the entire foot during the step and is described by [21] as:

$$
G R F_{T}[T]=\frac{1}{S} \sum_{i=1}^{S}\left(\sum_{t=0}^{T}\left(S_{i}[t]\right)\right),
$$

where $S$ is the number of pressure sensors in the apparatus, $i$ an individual sensor and $S_{i}[t]$ the output of sensor $i$ at a given time point $t$. Less commonly used profiles include the spatial average $\left(\mathrm{S}_{\text {ave }}\right)$, averaging raw sensor data across a mat, removing the spatial domain, $S_{\text {ave }}[t]=\frac{1}{S} \sum_{i=1}^{S}\left(S_{i}[t]\right)$ and the contour profiles, which provide the max $\left(\mathrm{S}_{u p}\right)$ and $\min$ $\left(\mathrm{S}_{l o}\right)$ amplitude values across all sensors respectively, $S_{u p}[t]=$ $\max _{i=1}^{S}\left(S_{i}[t]\right), S_{l o}[t]=\min _{i=1}^{S}\left(S_{i}[t]\right)$. All profiles provide a representation of the raw pressure data and were included in our geometric feature set.

The maximum, minimum and mean amplitude values and standard deviation (SD) amongst amplitude values were extracted from the raw data and geometric profiles to establish the statistical features. These statistical features provide representation of energy behaviour in force exerted during each footstep instance. The raw data statistics were drawn for each sensor in the apparatus and appended, resulting in a $1 \times 352$ feature vector for each foot that retained spatial information from the footstep profile. Statistical features were drawn from the GRF, $S_{\text {ave }}$ and contour profiles, giving a $1 \times 4$ feature vector per foot per profile. The finalised statistical portion of the geometric feature vector was an appending of these values to give a $1 \times 736$ feature vector.

The GRF profile was used to produce a series of step length features, derived from multiple varying starting thresholds. Step length was defined as the number of time samples between start and stop points determined by crossing a threshold value, with amplitude thresholds selected at 1, 2, 3,4 and $5 \times 10^{5}$. The final multi-scale geometric feature set was constructed using the statistical features and GRF step lengths taken from all 5 thresholds. By considering all 5 step length thresholds we aim to interpret the amplitude gradient of the beginning and end of the GRF profile, with closer step length values indicating a higher acceleration/deceleration in amplitude change and steeper gradient.

\section{Wavelet features}

Pressure signals present within footstep samples are a finite waveform, with a foot strike creating an increased amplitude reading until the foot is lifted, upon which the apparatus returns to a resting level. This temporally significant signal event lends itself to wavelet analysis methods such as CWT, allowing events to be studied in the time/scale/magnitude domain. CWT uses a given wavelet as comparison against the input signal within a given footstep signal, this comparison is repeated across temporal shifting and frequency scaling of the wavelet to return a coefficient matrix which represents our original input signal, allowing features describing the original raw data signal to be drawn.

The CWT coefficient matrix $C(s, p)$ is dependant upon the chosen mother wavelet, scaling and temporal positioning described as:

$$
C(s, p ; f(t), \Psi(t))=\int_{-\infty}^{\infty} f(t) \frac{1}{\sqrt{s}} \Psi *\left(\frac{t-p}{s}\right) d t,
$$

where the wavelet transform coefficient, $C$, is dependant on the chosen mother wavelet, $\Psi$, wavelet scaling, $s$, and temporal position along the original input time domain, $p$. For footstep analysis, CWT was performed using seven mother wavelets; Daubechies 2-6, Mexican Hat and Morlet. Wavelets were selected to cover a representative spread of waveforms, evaluating each wavelet's suitability in analysis of footstep pressure signals; db2-4 showing similarity to raw footstep signals and Mexican Hat providing representation of more general impulse signals. Using 8 scales allowed identification of signal events while maintaining a short feature vector size, saving classifier training time and lowering risk of over-fitting the classifier to observed data.

Footstep GRF profiles were subjected to CWT analysis with each of the above wavelets at 8 scales, returning an $S \times T$ coefficient matrix, where $T$ is the time samples within the raw footstep channel and $S$ the number of scales. Max, min, mean and SD were drawn from each scale of the coefficient matrix, removing the time domain, these values were appended to give the $1 \times 64$ GRF wavelet analysis feature vector.

\section{Proposed feature set}

Foot placement can impart varying pressure at localised regions across a surface; notably the first metatarsal head and heel provide high regions of pressure, while the shape of the foot is silhouetted on the surface it impacts [6], [7], [9]. The use of spatially reduced profiles is common within the literature, [7], [8], [10]-[12], while analysis of the spatial information contained within the raw pressure arrays is lacking. As such, we propose retention of spatial domain information by performing wavelet analysis on raw data as opposed to 
representative profiles such as the GRF. The intent is to provide training vectors that conserve spatio-temporal information that reflects the dispersal of pressure across the mat, which may be lost during the production of the GRF profile. To achieve this, CWT was carried out on raw signals of each sensor, using the above mother wavelets, returning an $S \times T \times 88$ coefficient matrix. For each sensor the max, min, mean and SD were drawn from each scale, removing $T$, leaving an $1 \mathrm{x} S$ vector per stat, per sensor, per foot. These were appended to produce the final 1x5632 wavelet feature vector for the footstep, retaining the spatial domain information of the footstep signal.

\section{E. Classifier construction and testing}

A Random Forest classifier was built to predict individual owners of a footstep due to the strength of the algorithm in handling large feature vectors during class partitioning and in training strong classifiers with minimal tuning [22], [23]. The dataset was split for training and testing using 10-fold cross validation partitioning, where the training set consisted of 9 folds and the $10^{t h}$ fold was the testing set. Forests were grown at densities of 10, 25, 50, 100, 200, 500 and 1000 trees per forest, providing insight into the trade-off between time and accuracy when increasing forest density. The number of samples used to split each node in the tree was set to the default value of the floored square root of $M$, the length of the feature vector used in an $N \times M$ feature set, a pre-tuning start point for exploratory analysis of feature sets. During RF construction the internally determined out-ofbag error estimate (OOB) method [22], despite negating the need for a dedicated training set, was applied to each fold in cross validation, allowing the classifier to be trained using a bootstrap sample of each training fold.

Passing test inputs through constructed forests returns a prediction of respective class label, taken via a vote from all trees within the forest. Once the entire testing set is classified, predictions are compared against ground truths and predictive error rate (PER) is returned as probability of incorrect prediction. Each replicate consisted of 10 PER values, one for each fold in the cross validation; with experiments carried out in triplicate. PERs were then averaged to determine partitioning error.

\section{RESUlT AND DiscusSiON}

Findings of the study are given below, detailing PERs returned by each classifier during 10 -fold cross validation testing. The geometric, GRF wavelet analysis and proposed feature set predictive errors are summarised in Fig.2 and Tab.II.

\section{A. Forest density}

In all cases, increase in forest density led to increased predictive accuracy as expected, plateauing once tree density exceeded 500 trees in the majority of cases. Increase in forest density also increased computational time and resource required for model construction, with marginal performance benefit post-plateau. For consistency, from this point on we will only discuss PERs obtained using 1000 trees.
TABLE II: Summary of PERs (\% error). 10 tree forest results are omitted due to lack of space.

\begin{tabular}{|c|c|c|c|c|c|c|}
\hline \multirow[b]{2}{*}{ Feature Set } & \multicolumn{6}{|c|}{ Density of Forest (number of trees) } \\
\hline & 25 & 50 & 100 & 200 & 500 & 1000 \\
\hline \multicolumn{7}{|c|}{ Geometric Features } \\
\hline $\begin{array}{l}\text { Geometric statis- } \\
\text { tics only }\end{array}$ & $\begin{array}{l}32.2 \\
\pm 1.2\end{array}$ & $\begin{array}{l}29.4 \\
\pm 0.9\end{array}$ & $\begin{array}{l}27.9 \\
\pm 1.1\end{array}$ & $\begin{array}{l}27.0 \\
\pm 1.0\end{array}$ & $\begin{array}{l}26.5 \\
\pm 0.9\end{array}$ & $\begin{array}{l}26.4 \\
\pm 1.0\end{array}$ \\
\hline $\begin{array}{l}\text { Geometric }+ \text { Step } \\
\text { length } 1 \times 10^{5}\end{array}$ & $\begin{array}{l}32.5 \\
\pm 1.5\end{array}$ & $\begin{array}{l}29.2 \\
\pm 1.4\end{array}$ & $\begin{array}{l}27.6 \\
\pm 1.1\end{array}$ & $\begin{array}{l}27.0 \\
\pm 1.3\end{array}$ & $\begin{array}{l}26.5 \\
\pm 1.4\end{array}$ & $\begin{array}{l}25.8 \\
\pm 1.5\end{array}$ \\
\hline $\begin{array}{l}\text { Geometric + Step } \\
\text { length } 2 \times 10^{5}\end{array}$ & $\begin{array}{l}32.4 \\
\pm 1.5\end{array}$ & $\begin{array}{l}29.2 \\
\pm 1.6\end{array}$ & $\begin{array}{l}27.5 \\
\pm 1.8\end{array}$ & $\begin{array}{l}27.4 \\
\pm 1.8\end{array}$ & $\begin{array}{l}26.7 \\
\pm 1.1\end{array}$ & $\begin{array}{l}26.6 \\
\pm 1.7\end{array}$ \\
\hline $\begin{array}{l}\text { Geometric + Step } \\
\text { length } 3 \times 10^{5}\end{array}$ & $\begin{array}{l}31.8 \\
\pm 1.4\end{array}$ & $\begin{array}{l}29.2 \\
\pm 1.2\end{array}$ & $\begin{array}{l}27.6 \\
\pm 1.1\end{array}$ & $\begin{array}{l}26.8 \\
\pm 1.2\end{array}$ & $\begin{array}{l}26.5 \\
\pm 1.3\end{array}$ & $\begin{array}{l}26.3 \\
\pm 1.2\end{array}$ \\
\hline $\begin{array}{l}\text { Geometric }+ \text { Step } \\
\text { length } 4 \times 10^{5}\end{array}$ & $\begin{array}{l}32.3 \\
\pm 1.3\end{array}$ & $\begin{array}{l}29.1 \\
\pm 1.2\end{array}$ & $\begin{array}{l}27.5 \\
\pm 1.1\end{array}$ & $\begin{array}{l}26.7 \\
\pm 1.0\end{array}$ & $\begin{array}{l}26.4 \\
\pm 0.9\end{array}$ & $\begin{array}{l}26.1 \\
\pm 1.0\end{array}$ \\
\hline $\begin{array}{l}\text { Geometric }+ \text { Step } \\
\text { length } 5 \times 10^{5}\end{array}$ & $\begin{array}{l}32.0 \\
\pm 1.4\end{array}$ & $\begin{array}{l}29.0 \\
\pm 1.2\end{array}$ & $\begin{array}{l}27.5 \\
\pm 1.0\end{array}$ & $\begin{array}{l}26.7 \\
\pm 0.9\end{array}$ & $\begin{array}{l}26.2 \\
\pm 0.8\end{array}$ & $\begin{array}{l}26.1 \\
\pm 0.7\end{array}$ \\
\hline $\begin{array}{l}\text { Finalised } \\
\text { Geometric }\end{array}$ & $\begin{array}{l}32.0 \\
\pm 1.0\end{array}$ & $\begin{array}{l}28.6 \\
\pm 1.2\end{array}$ & $\begin{array}{l}27.2 \\
\pm 1.0\end{array}$ & $\begin{array}{l}26.4 \\
\pm 0.9\end{array}$ & $\begin{array}{l}25.8 \\
\pm 1.0\end{array}$ & $\begin{array}{l}25.5 \\
\pm 1.2\end{array}$ \\
\hline \multicolumn{7}{|c|}{ GRF CWT Features } \\
\hline GRF CWT & $\begin{array}{l}66.8 \\
\pm 1.0\end{array}$ & $\begin{array}{l}64.5 \\
\pm 1.2\end{array}$ & $\begin{array}{l}63.3 \\
\pm 1.2\end{array}$ & $\begin{array}{l}62.5 \\
\pm 1.0\end{array}$ & $\begin{array}{l}61.7 \\
\pm 1.1\end{array}$ & $\begin{array}{l}61.3 \\
\pm 1.3\end{array}$ \\
\hline $\begin{array}{l}\text { Geometric } \\
\text { GRF CWT }\end{array}$ & $\begin{array}{l}31.7 \\
\pm 1.2\end{array}$ & $\begin{array}{l}28.9 \\
\pm 1.3\end{array}$ & $\begin{array}{l}26.6 \\
\pm 1.2\end{array}$ & $\begin{array}{l}26.3 \\
\pm 1.3\end{array}$ & $\begin{array}{l}25.6 \\
\pm 1.3\end{array}$ & $\begin{array}{l}25.4 \\
\pm 1.4\end{array}$ \\
\hline \multicolumn{7}{|c|}{ Proposed: Spatially Retentive CWT Features } \\
\hline Spatial CWT & $\begin{array}{l}21.9 \\
\pm 1.1\end{array}$ & $\begin{array}{l}19.2 \\
\pm 1.2\end{array}$ & $\begin{array}{l}18.3 \\
\pm 1.4\end{array}$ & $\begin{array}{l}17.5 \\
\pm 1.4\end{array}$ & $\begin{array}{l}17.0 \\
\pm 1.5\end{array}$ & $\begin{array}{l}17.0 \\
\pm 1.2\end{array}$ \\
\hline $\begin{array}{l}\text { Geometric + Spa- } \\
\text { tial CWT }\end{array}$ & $\begin{array}{l}22.2 \\
\pm 1.7\end{array}$ & $\begin{array}{l}19.6 \\
\pm 1.1\end{array}$ & $\begin{array}{l}18.4 \\
\pm 1.5\end{array}$ & $\begin{array}{l}17.6 \\
\pm 1.1\end{array}$ & $\begin{array}{l}17.1 \\
\pm 1.1\end{array}$ & $\begin{array}{l}16.7 \\
\pm 1.2\end{array}$ \\
\hline $\begin{array}{l}\text { Spatial CWT + } \\
\text { GRF Wavelet }\end{array}$ & $\begin{array}{l}31.3 \\
\pm 1.5\end{array}$ & $\begin{array}{l}28.5 \\
\pm 1.2\end{array}$ & $\begin{array}{l}26.2 \\
\pm 1.2\end{array}$ & $\begin{array}{l}26.1 \\
\pm 1.4\end{array}$ & $\begin{array}{l}25.3 \\
\pm 1.3\end{array}$ & $\begin{array}{l}25.0 \\
\pm 1.3\end{array}$ \\
\hline $\begin{array}{ll}\text { Geometric } & + \\
\text { GRF CWT } & + \\
\text { Spatial CWT }\end{array}$ & $\begin{array}{l}\mathbf{2 1 . 7} \\
\pm \mathbf{0 . 9}\end{array}$ & $\begin{array}{l}19.2 \\
\pm 1.3\end{array}$ & $\begin{array}{l}18.1 \\
\pm 1.1\end{array}$ & $\begin{array}{l}17.6 \\
\pm 1.2\end{array}$ & $\begin{array}{l}\mathbf{1 6 . 8} \\
\pm 0.7\end{array}$ & $\begin{array}{l}\mathbf{1 6 . 3} \\
\pm 0.7\end{array}$ \\
\hline
\end{tabular}

\section{B. Geometric features}

Using only statistical features derived from raw data and representative profiles it was possible to achieve average PERs as low as $26.4 \% \pm 1.0 \%$. There was high variability between the importance of each sensor within the apparatus; this can be expected when a foot only strikes a given number of sensors on the mat, suggesting that orientation or normalisation is required to directly compare the same sensor in two samples.

GRF step length as a solitary feature was omitted due to it's weak ability as a classifier, providing PERs of $>80 \%$, most likely due to inherent problems in using a singular, highly variable feature to describe partitions between classes. Using individual scale GRF step lengths combined with geometric statistic features there was a marginal average decrease in PER of $0.5 \%$ when using thresholds set at $1,3,4$ and $5 \times 10^{5}$, with marginal increase in PER when the threshold was set to $2 \times 10^{5}$.

The finalised geometric feature set, containing the statistical features and the step lengths determined at all 5 thresholds, provided PERs of $25.5 \% \pm 1.2 \%$, Fig.2- solid line, showing that a Random Forest classifier is able to partition weak pressure based footstep data with even the crudest of feature 


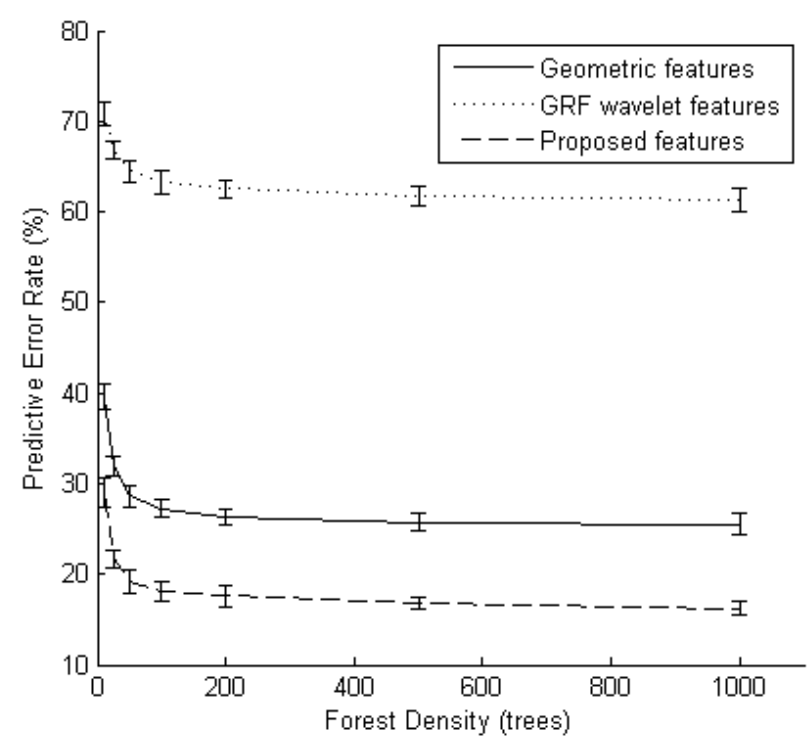

Fig. 2: Impact of key feature sets on Predictive Error Rate.

sets. This classification accuracy, from a dataset of 94 class labels, allows us to have confidence in predictive capabilities of the classifier, suggesting use of geometrical features can provide strong partitioning for individual classification and, with further feature set optimisation, can lead to adoption of footsteps as an identifier.

\section{Wavelet analysis of the GRF profile}

Wavelet analysis of the spatially reduced GRF profile provided weak classification; with the best performing wavelet being the Mexican Hat wavelet, producing PERs of $61.3 \% \pm 1.3 \%$, Fig.2- dotted line. When these features were appended with the geometrical feature set there was an improved accuracy to $25.4 \% \pm 1.4 \%$, a marginal improvement over solitary use of the geometric feature set. The wavelet analysis of the GRF profile provided poor partitioning of our dataset, with PERs falling well below those of the geometric feature set.

It is believed that over-simplification of footstep information resulted in poor fitting of the classifier to the training data and thus the classifier struggles when introduced to new input samples. It may be worthwhile selecting more distinct features from the wavelet analysis of GRF profiles, with more descriptive features perhaps providing higher partitioning accuracy, however with our statistical features we found that the loss of the spatial domain produces weak label partitions.

\section{Proposed feature set}

Wavelet analysis of raw input signals provided footstep event localisation in the scale/time domain at 8 scales and these wavelet features greatly improved prediction accuracy. Observed PERs fell to PER of $17.0 \% \pm 1.2 \%$ when using the Mexican Hat mother wavelet, the strongest class partitioning of all wavelets evaluated. Combining spatially retentive wavelet analysis with geometric feature set provided PERs of $16.7 \% \pm 1.2 \%$, a further increase to accuracy. These results show that the inclusion of the spatial domain information provides a benefit in identifying the individual participants within the dataset. There is an increase in accuracy of nearly $9 \%$ when compared to the geometric features and over $44 \%$ increase in accuracy compared to the wavelet features extracted from the GRF profile, clearly showing the importance of the spatial domain.

\section{E. Feature set aggregation}

By appending all three suggested feature sets; geometric, GRF and spatially retentive wavelet features, we were able to obtain PERs of $16.3 \% \pm 0.7 \%$ using the Mexican Hat wavelet for both sets of wavelet analysis, Fig.2- dashed line. This further increase in accuracy despite the increase in partitioning variables is due to the ability of the Random Forest algorithm to identify the most important variables in the partitioning of the class labels; separating them out from the weaker features [22], [23]. The trade-off between the extraction of this larger feature set and the small increase in accuracy may suggest omitting the GRF wavelet features in favour of utilising the geometric and spatially retentive feature set combination.

We conclude that use of CWT for analysis of footstep pressure profiles is most effective when considering the spatial domain of the problem, most notably the raw data shown with the raw pressure signals. The dataset contained no limitations on the footwear used and variable participant shoe size and morphology, this suggests that there should spatial information with the data that can be used as a partitioning parameter for classification. The compression of the spatial domain from the signals in the production of the GRF profile is believed to have lost partitioning information from the realistic dataset and as such we recommend the use of wavelet analysis on raw signals to provide improved accuracy.

\section{Closing Remarks}

This study has focused on using a distinctly weak biometric in a classification problem in contrast to verification, in which the system is predicting sample class from multiple labels, instead of attempting to accept or reject a given sample as an enrolled user. This highly variable biometric will rarely reach the accuracy rates of established biometrics; despite this, we have been able to use simple geometric features to produce a classifier able to predict footstep owners from a large, diverse and realistic dataset. The least impact to class partitioning comes from mean values for each raw data channel, which could be expected from a finite input signal with a rest state of zero, in which averaging would lose key temporal events.

We believe that the classification results show the importance of considering the spatial information of a footstep, a critical parameter that should not be overlooked when using footsteps to identify individuals. Use of wavelet analysis on raw pressure signals improved prediction accuracy over that of spatially reduced profile features, while removing the spatial domain via production of the GRF profile led to wavelet analysis with weakened predictive capabilities. Therefore, as 
expected, the biometric information within a footstep is a combination of temporally and spatially important events; this suggests use of feature sets that represent the spatio-temporal domain as a whole is of benefit to classifier construction and dataset partitioning.

Feature impact on node splits have shown that not every sensor is providing equal benefit to dataset partitioning, however all sensors were included due to lack of footstep orientation pre-processing. Orientation may allow more informative comparison between footsteps. Further work will explore the extraction of detailed features from wavelet coefficients and the production of dedicated footstep signal wavelets.

\section{REFERENCES}

[1] F. Besbes, H. Trichili, B. Solaiman, "Multi-modal biometric system based on fingerprint identification and iris recognition," in Information and Communication Technologies: From Theory to Applications, 2008.

[2] S. A. Vivek, J. Aravinth and S. Valarmathy, "Feature extraction for multi-modal biometric and study of fusion using Gaussian mixture model," in Pattern Recognition, Informatics and Medical Engineering, 2012.

[3] B. Daubney, X. Xie, "Tracking 3D human pose with large root node uncertainty", in IEEE Conference on Computer Vision and Pattern Recognition, pp. 1321-1328, 2011.

[4] J. Deng, X. Xie and B. Daubney, "A bag of words approach to subject specific 3D human pose interaction classification with random decision forests", in Graphical Models, vol. 76, no. 3pp. 162-171, 2014.

[5] S. Dahel and Q. Xiao, "Accuracy performance analysis of multi-modal biometrics," in Information Assurance Workshop. IEEE SMC, 2003, pp. 170-173.

[6] K. Nagwanshi and S. Dubey, "Biometric authentication using human footprint," Int. J. Appl. Inform. Syst., vol. 3, no. 7, pp. $1-6,2012$.

[7] S. P. Moustakidis, J. B. Theocharis and G. Giakas, "Subject recognition based on ground reaction force measurements of gait signals," in IEEE, T-SMC B, Cybern., vol. 38, no. 6, pp. 1476-1485, 2008.

[8] R. J. Orr and G. D. Abowd, "The Smart Floor: a mechanism for natural user identification and tracking," in CHI Extended Abstracts Human Factors in Computing Systems, 2000, pp. 275-276.

[9] H. Elftman, "Forces and energy changes in the legs during walking," Am. J. Physiol., vol. 125, no. 2, pp. 339-356, 1939.

[10] M. Addlesee, A. Jones, F. Livesey and F. Samaria, "The ORL Active Floor," IEEE Pers. Commun., vol. 4, no. 5, pp. 35-41, 1997.

[11] J. Suutala and J. Röning, "Methods for person identification on a pressure-sensitive floor: experiments with multiple classifiers and reject option," Information Fusion, vol. 9 , no. 1, pp. 21-40, Jan. 2008.

[12] S. Pirttikangas, J. Suutala, J. Riekki, J. Röning, ”Learning Vector Quantization in Footstep Identification," in Int. Conf. Artificial Intelligence and Applications, 2003, pp. 413-417.

[13] P. M. Forsman, E. M. Topplia and E. O. Hæggström, "Wavelet analysis to detect gait events," in IEEE EMBS, 2009.

[14] A. Itai and H. Yasukawa, "Personal Identification using Footstep Based on Wavelets," in Int. Symp. Intelligent Signal Processing and Communication Systems, 2006, pp. 383-386.

[15] A. Itai and H. Yasukawa, "Footstep Classification using Wavelet Decomposition," in Int. Symp. Communications and Information Technologies, 2007, pp. 551-556.
[16] H. Xing, F. Li and Y.Liu, "Wavelet Denoising and Feature Extraction of Seismic Signal fot Footstep Detection," in Int. Conf. Wavelet Analysis and Pattern Re-cognition, 2007, pp. 218-223.

[17] V. V. Reddy, V. Divya, A. W. H. Khong and B. P. Ng, "Footstep Detection and Denoising using a Single Triaxial Geophone," in Asia Pacific Conf. on Circuits and Systems, 2010, pp. 11711174 .

[18] A. Mostayed, S. Kim, M. M. G. Mazumder and S.J. Park, "Foot Step Based Person Identification using Histogram Similarity and Wavelet Decomposition," in Int. Conf. Information Security and Assurance, 2008, pp. 307-311.

[19] F. Verdini, T. Leo, S. Fioretti, M. G. Benedetti, F. Catani and S. Giannini, "Analysis of Ground Reaction Forces by means of Wavelet Transform,"Clinical Biomechanics", pp. 607-610, 2000.

[20] R. Vera-Rodriguez, R. P. Lewis, J. S. D. Mason, N. W. D. Evans, "A large scale footstep database for biometric studies created using cross-biometrics for labelling," in Int. Conf. Control, Automation, Robotics and Vision, 2008, pp. 13611366.

[21] R. Vera-Rodriguez, J. S. D. Mason, J. Fierrez and J. OrtegaGarcia, "Analysis of time domain information for footstep recognition," Advances in Visual Computing, pp. 489-498, 2010.

[22] L. Breiman, "Random Forests," Machine Learning, pp. 5-32, 2001.

[23] G. Biau, "Analysis of a Random Forest Model," Machine Learning Research, pp. 1063-1095, 2012. 